\title{
Dynamical Reduction Models with General Gaussian Noises
}

\author{
Angelo Bassi* \\ The Abdus Salam International Centre for Theoretical Physics, Trieste, Italy, \\ and \\ GianCarlo Ghirardi ${ }^{\dagger}$ \\ Department of Theoretical Physics of the University of Trieste, and \\ the Abdus Salam International Centre for Theoretical Physics, Trieste, Italy.
}

\begin{abstract}
We consider the effect of replacing in stochastic differential equations leading to the dynamical collapse of the statevector, white noise stochastic processes with non white ones. We prove that such a modification can be consistently performed without altering the most interesting features of the previous models. One of the reasons to discuss this matter derives from the desire of being allowed to deal with physical stochastic fields, such as the gravitational one, which cannot give rise to white noises. From our point of view the most relevant motivation for the approach we propose here derives from the fact that in relativistic models the occurrence of white noises is the main responsible for the appearance of untractable divergences. Therefore, one can hope that resorting to non white noises one can overcome such a difficulty. We investigate stochastic equations with non white noises, we discuss their reduction properties and their physical implications. Our analysis has a precise interest not only for the above mentioned subject but also for the general study of dissipative systems and decoherence.
\end{abstract}

\section{Introduction}

The aim of dynamical reduction models [1]-[7] is to combine the Schrödinger evolution and the wavepacket reduction postulate into one universal dynamical equation, which is assumed to govern all physical processes. In this way, such a dynamics accounts both for the quantum properties of microscopic systems and for the classical properties of macroscopic ones.

*e-mail: bassi@ictp.trieste.it

†e-mail: ghirardi@ts.infn.it 
This goal is achieved by adding to the Schrödinger equation new stochastic terms which induce a diffusion process - guided by a set of Gaussian white noises - of the statevector in Hilbert space: it is precisely this sort of "random walk" which is responsible for the localization mechanism. We will review these attempts in section 2 .

The main aim of this paper is to generalize the basic equations of dynamical reduction models to the case of general Gaussian noises, not necessarily white in time. There are three reasons for considering models of this kind: first of all, it is interesting to analyze if, and to which extent, the nice features of the reduction mechanisms proposed up to now depend on the white-noise character of the stochastic processes. Secondly, it has been argued several times that the stochastic processes should be related to physical fields - the most promising being the gravitational field; in such a case, the stochastic processes cannot be white, since white noises are never realized in nature. Finally, the third motivation to look for generalizations of the previous models has to do with the important problem of working out relativistic models of wavepacket reduction 四. In fact, even though it has been proved that models of this type share all the nice features of the non relativistic ones, they suffer from the serious drawback of inducing an infinite increase of the energy of physical systems. This infinite increase is basically caused by the local coupling between the quantum fields and the white-noise stochastic fields appearing in such theories. Accordingly, a reasonable way to overcome such a difficulty is to replace the white-noise fields with more general ones: this will be the subject of papers in preparation. Such attempts, however, require a preliminary and detailed investigation aimed to clarify that the consideration of noises which are non white in time does not lead to inconsistencies and preserves the nice features of the models based on noises which are white. The analysis we are going to perform is also of interest, per se, for the study of stochastic dynamical equations in general, a subjet which has received a lot of attention in recent times.

Sections 3 to 6 of this paper are devoted to a general discussion of nonwhite random differential equations in Hilbert space. We will show that the most important features of white-noise stochastic equations, in particular the desired reduction effects, hold also in the more general case, thus proving that the white-noise character of the stochastic processes is not an essential element of the dynamical reduction program. Dynamical reduction models based on general Gaussian noises have been studied by Pearle [8, 9] and Diósi et al [10, 11] along different lines. In the final section of the paper, we apply the results and 
the formalism of the previous sections to a specific model of dynamical reduction and we prove that it leads precisely to the localization of macroscopic objects in space.

\section{Review of CSL}

The CSL (Continuous Spontaneous Localizations) version of Dynamical Reduction Models [2, 3] is based on a stochastic Schrödinger equation which, in the Stratonovich language, takes the form:

$$
\frac{d|\psi(t)\rangle}{d t}=\left[-\frac{i}{\hbar} H_{0}+\sum_{i} A_{i} w_{i}(t)-\gamma \sum_{i} A_{i}^{2}\right]|\psi(t)\rangle .
$$

Here, $H_{0}$ is the free Hamiltonian of the system; $\left\{A_{i}\right\}$ is a set of commuting selfadjoint operators (representing the preferred basis) whose common eigenmanifolds are the linear manifolds into which the statevectors of individual physical systems are driven; $w_{i}(t)$ are $c$-number independent stochastic processes with a gaussian distribution which is white in time:

$$
\left\langle\left\langle w_{i}(t)\right\rangle\right\rangle=0, \quad\left\langle\left\langle w_{i}\left(t_{1}\right) w_{j}\left(t_{2}\right)\right\rangle\right\rangle=\gamma \delta_{i j} \delta\left(t_{1}-t_{2}\right),
$$

the symbol $\langle\langle\rangle$.$\rangle denoting the stochastic average associated to the process (2.2).$ Equation (2.1) describes a diffusion process in Hilbert space; it is a linear equation like the Schrödinger equation, but it does not preserve the norm of $|\psi(t)\rangle$ since the evolution is not unitary, due to the presence of the last two terms on the right hand side. The solution $|\psi(t)\rangle$ cannot therefore be endowed with a direct physical meaning.

To overcome this difficulty, and at the same time to ensure that the reduction mechanism reproduces the quantum mechanical probabilities, the following strategy has been adopted [2, 3]. The physical vectors are the normalized solutions of equation (2.1):

$$
\left|\psi_{\text {Phys }}(t)\right\rangle=\frac{|\psi(t)\rangle}{\||\psi(t)\rangle \|},
$$

and it is assumed that any particular realization of the stochastic processes $w_{i}(t)$, yielding the state $\left|\psi_{\text {phys }}(t)\right\rangle$, has a probability of occurrence $P_{\text {Cook }}[w(t)]$ equal to:

$$
P_{\text {Cook }}[w(t)]=P_{\text {Raw }}[w(t)] \||\psi(t)\rangle \|^{2},
$$

where $P_{\text {Raw }}[w(t)]$ is the original probability distribution of the gaussian white noises given by (2.2). 
Of course, since (2.4) defines a probability distribution, it must sum to 1:

$$
\begin{aligned}
\int \mathcal{D}[w(t)] P_{\text {Cook }}[w(t)] & =1 \\
& =\int \mathcal{D}[w(t)] P_{\text {Raw }}[w(t)] \||\psi(t)\rangle \|^{2} \\
& =\langle\langle\langle\psi(t) \mid \psi(t)\rangle\rangle\rangle
\end{aligned}
$$

(we remember that $\langle\langle\rangle$.$\rangle refers to the average with respect to the original prob-$ ability distribution $\left.P_{\text {Raw }}[w(t)]\right)$. Equation (2.5) imposes that the stochastic average of the square norm of the vector $|\psi(t)\rangle$ be conserved; as it can be easily verified, equation (2.1) guarantees that this is the case.

It is possible to write [3 the norm-preserving equation for the physical vector $\left|\psi_{\text {Phys }}(t)\right\rangle$ which is equivalent to eq. (2.1) with the prescription (2.4): such an equation is nonlinear and more difficult to handle. We will not consider it here.

In reference [3] it has been shown that, if one ignores the Hamiltonian term $H_{0}$, equation (2.1) together with the cooking prescription (2.4) — or, alternatively, the corresponding norm-preserving equation - drives the statevector of any individual physical system into one of the common eigenmanifolds of the operators $A_{i}$. Here we will simply show that equation (2.1) implies the diagonalization of the density matrix with respect to the basis of the common eigenmanifolds of the operators $A_{i}$.

The statistical operator is the average value, with respect to the the "cooked" (i.e. the physical) probability distribution $P_{\mathrm{Cook}}[w(t)]$, of the projections operators onto the one dimensional linear manifolds spanned by the physical vectors $\left|\psi_{\text {Phys }}(t)\right\rangle$ :

$$
\begin{aligned}
\rho(t) & =\int \mathcal{D}[w(t)]\left|\psi_{\text {Phys }}(t)\right\rangle\left\langle\psi_{\text {Phys }}(t)\right| P_{\text {Cook }}[w(t)] \\
& =\langle\langle\mid \psi(t)\rangle\langle\psi(t) \mid\rangle\rangle .
\end{aligned}
$$

We note that, from the mathematical point of view, $\rho(t)$ corresponds also to the ensemble of operators $|\psi(t)\rangle\langle\psi(t)|(|\psi(t)\rangle$ being non normalized), averaged with the raw probability distribution $P_{\text {Raw }}[w(t)]$. Thanks to this property the dynamical evolution equation for $\rho(t)$ can be easily derived:

$$
\frac{d \rho(t)}{d t}=-\frac{i}{\hbar}\left[H_{0}, \rho(t)\right]-\frac{\gamma}{2} \sum_{i}\left[A_{i},\left[A_{i}, \rho(t)\right]\right] .
$$

\footnotetext{
${ }^{1}$ Of course, the diagonalization of the density matrix is only a necessary not a sufficient condition for the localizations to occur, as it has been shown jn_reference [12]. Anyway, as already remarked, reference [3] contains a proof that equation (2.1) does imply the reduction of the statevector into the desired eigenmanifolds.
} 
To show the effect of the reducing terms, let us suppose, for simplicity, that the common eigenmanifolds $M_{\alpha}$ of the operators $A_{i}$ (which we assume to have a purely discrete spectrum) are one-dimensional and we call $|\alpha\rangle$ the vector spanning $M_{\alpha}$ :

$$
A_{i}|\alpha\rangle=a_{i \alpha}|\alpha\rangle .
$$

For the moment, let us ignore the Hamiltonian term $H_{0}$. Then, equation (2.7) implies the following equation for the matrix elements $\langle\alpha|\rho(t)| \beta\rangle$ :

$$
\frac{d\langle\alpha|\rho(t)| \beta\rangle}{d t}=-\frac{\gamma}{2} \sum_{i}\left(a_{i \alpha}-a_{i \beta}\right)^{2}\langle\alpha|\rho(t)| \beta\rangle .
$$

Equation (2.9) shows that the off-diagonal elements of the density matrix, corresponding to the interference terms arising from the superpositions of different eigenstates of $A_{i}$, are exponentially damped. The diagonal elements, on the other hand, do not change with time.

Up to now we have described the general formal structure of CSL. To give a physical content to the model, one must choose the "preferred basis", i.e. the operators $A_{i}$ which define the manifolds onto which the wavefunction is reduced. Obviously, our aim is to induce the spatial localization of macroscopic objects. To this purpose one can make the choice [3]:

$$
A_{i} \longrightarrow \mathcal{N}(\mathbf{x})=\left(\frac{\alpha}{2 \pi}\right)^{\frac{3}{2}} \sum_{s} \int d^{3} y e^{-\frac{\alpha}{2}(\mathbf{x}-\mathbf{y})^{2}} a^{\dagger}(\mathbf{y}, s) a(\mathbf{y}, s),
$$

where $a^{\dagger}(\mathbf{y}, s)$ and $a(\mathbf{y}, s)$ are the creation and annihilation operators for a constituent with spin component $s$, at point $\mathrm{y}$. The parameter $1 / \sqrt{\alpha}$ is a measure the localization accuracy of the reducing mechanism: for physical reasons [1] its value has been chosen to be $1 / \sqrt{\alpha} \simeq 10^{-5} \mathrm{~cm}$. The value of the other parameter of the theory, $\gamma$, which measures the strength of the correlation function of the white noises, is related to the parameter $\lambda \simeq 10^{-16} \mathrm{sec}^{-1}$ of QMSL 1], which, within such a discrete model, specifies the frequency of the random reduction processes, according to $\gamma=\lambda(4 \pi / \alpha)^{3 / 2}$. Accordingly, the stochastic processes $w_{i}(t)$ are replaced by a gaussian stochastic field $w(\mathbf{x}, t)$, whose first two moments are:

$$
\left\langle\langle w(\mathbf{x}, t)\rangle=0, \quad\left\langle\left\langle w\left(\mathbf{x}, t_{1}\right) w\left(\mathbf{y}, t_{2}\right)\right\rangle\right\rangle=\gamma \delta(\mathbf{x}-\mathbf{y}) \delta\left(t_{1}-t_{2}\right) .\right.
$$

${ }^{2}$ If more than one type of particle is involved, an extra sum over the different kinds of particles must appear in the definition of $\mathcal{N}(\mathbf{x})$. 
The modified Schrödinger equation (2.1) becomes then:

$$
\frac{d|\psi(t)\rangle}{d t}=\left[-\frac{i}{\hbar} H_{0}+\int d^{3} x \mathcal{N}(\mathbf{x}) w(\mathbf{x}, t)-\gamma \int d^{3} x \mathcal{N}^{2}(\mathbf{x})\right]|\psi(t)\rangle,
$$

and the corresponding equation for the statistical operator is:

$$
\frac{d \rho(t)}{d t}=-\frac{i}{\hbar}\left[H_{0}, \rho(t)\right]-\frac{\gamma}{2} \int d^{3} x[\mathcal{N}(\mathbf{x}),[\mathcal{N}(\mathbf{x}), \rho(t)]] .
$$

Equations (2.12) and (2.13) exhibit two basic features:

- At the microscopic level - i.e. when only few constituents are involved the new terms do not alter in any appreciable way the pure Schrödinger evolution: all quantum properties of micro-systems are left essentially unchanged. This is due to the fact that the value of $\lambda$ is extremely small.

- At the macroscopic level, on the other hand, the new terms induce in a very short time - much shorter than the perception time of a conscious observer - the suppression of the superposition of different macroscopic states and the reduction to one of them. Accordingly, macroscopic objects are always localized in space, and their classical properties are restored.

This is the way in which dynamical reduction models are able to account for the behaviour of both microscopic quantum and macroscopic classical systems.

\section{Dynamical reduction models with general Ga- ussian noises}

In this section we begin the analysis of dynamical reduction models in which the reduction mechanism is controlled by general Gaussian noises. The first task is to derive a modified Schrödinger equation generalizing equation (2.1), and preserving the average value of the square norm of vectors, so that the cooking prescription can be applied to it.

Let us then consider the following equation:

$$
\frac{d|\psi(t)\rangle}{d t}=\left[-\frac{i}{\hbar} H_{0}+\sum_{i} A_{i} w_{i}(t)\right]|\psi(t)\rangle,
$$

where, as before, $H_{0}$ is the Hamiltonian of the system, $\left\{A_{i}\right\}$ is a set of commuting self-adjoint operators, and $w_{i}(t)$ are $c$-number gaussian stochastic processes 
whose first two moments are 3 :

$$
\left\langle\left\langle w_{i}(t)\right\rangle\right\rangle=0, \quad\left\langle\left\langle w_{i}\left(t_{1}\right) w_{j}\left(t_{2}\right)\right\rangle\right\rangle=\gamma D_{i j}\left(t_{1}, t_{2}\right) .
$$

As in standard CSL, the evolution described by equation (3.1) is not unitary and it does not preserve the norm of the statevector; we then follow the same prescription outlined in section 2 . We consider as physical vectors the normalized ones:

$$
\left|\psi_{\text {Phys }}(t)\right\rangle=\frac{|\psi(t)\rangle}{\||\psi(t)\rangle \|},
$$

and we assume that any particular realization of the stochastic processes $w_{i}(t)$ has a probability of occurrence $P_{\text {Cook }}[w(t)]$ equal to:

$$
P_{\text {Cook }}[w(t)]=P_{\text {Raw }}[w(t)] \||\psi(t)\rangle \|^{2},
$$

where $P_{\text {Raw }}[w(t)]$ is now the gaussian probability distribution defined by (3.2). The above assumptions guarantee, as we will show in section 5 , that the reduction probabilities reproduce standard quantum mechanical probabilities.

As in section 2, we have to impose that equation (3.4) correctly defines a probability distribution, i.e. that it sums to 1 . From equation (2.5) we see that this is equivalent to requiring that the time derivative of $\langle\langle\langle\psi(t) \mid \psi(t)\rangle\rangle\rangle$ is zero. Let us evaluate it:

$$
\begin{aligned}
\frac{d}{d t}\langle\langle\langle\psi(t) \mid \psi(t)\rangle\rangle\rangle= & \left.\left\langle\left\langle\left[\frac{d\langle\psi(t)|}{d t}\right] \mid \psi(t)\right\rangle\right\rangle\right\rangle+\left\langle\left\langle\left\langle\psi(t) \mid\left[\frac{d|\psi(t)\rangle}{d t}\right]\right\rangle\right\rangle=\right. \\
= & \left\langle\left\langle\left\langle\psi(t)\left|\left[+\frac{i}{\hbar} H_{0}+\sum_{i} A_{i} w_{i}(t)\right]\right| \psi(t)\right\rangle\right\rangle\right\rangle+ \\
& \left\langle\left\langle\left\langle\psi(t)\left|\left[-\frac{i}{\hbar} H_{0}+\sum_{i} A_{i} w_{i}(t)\right]\right| \psi(t)\right\rangle\right\rangle\right\rangle .
\end{aligned}
$$

The two terms involving the Hamiltonian $H_{0}$ cancel out (in fact they describe the unitary part of the evolution); the noises $w_{i}(t)$, being $c$-numbers, can be taken out of the scalar product, so that:

$$
\frac{d}{d t}\langle\langle\langle\psi(t) \mid \psi(t)\rangle\rangle\rangle=2 \sum_{i}\left\langle\left\langle\left\langle\psi(t)\left|A_{i}\right| \psi(t)\right\rangle w_{i}(t)\right\rangle\right\rangle .
$$

The right hand side of (3.5) can be rewritten with the help of the FurutsuNovikov formula [16]:

$$
\left\langle\left\langle F[w(t)] w_{i}(t)\right\rangle\right\rangle=\gamma \sum_{j} \int_{0}^{+\infty} D_{i j}(t, s)\left\langle\left\langle\frac{\delta F[w(t)]}{\delta w_{j}(s)}\right\rangle\right\rangle d s
$$

\footnotetext{
${ }^{3}$ There is no loss of generality in considering gaussian processes with zero mean. In fact, if $\left\langle\left\langle w_{i}(t)\right\rangle\right\rangle=m_{i}(t) \neq 0$, we can always define new processes $z_{i}(t)=w_{i}(t)-m_{i}(t)$, which have zero mean, and rewrite the Schrödinger equation (3.1) in terms of the processes $z_{i}(t)$.
} 
(for simplicity, throughout this section we take $t_{0}=0$ as the initial time). $F[w(t)]$ is any functional of the stochastic fields $w_{i}(t)$; in the present case case, $F[w(t)]=\left\langle\psi(t)\left|A_{i}\right| \psi(t)\right\rangle$.

The formal solution of equation (3.1) is:

$$
|\psi(t)\rangle=T e^{-\frac{i}{\hbar} H_{0} t+\sum_{i} A_{i} \int_{0}^{t} w_{i}(s) d s}|\psi(0)\rangle .
$$

Note that, since $|\psi(t)\rangle$ depends on the stochastic processes $w_{i}(s)$ only within the time-interval $[0, t]$, the functional derivative of $|\psi(t)\rangle$ with respect to $w_{j}(s)$ is zero if $s \notin[0, t]$. We then have:

$$
\begin{aligned}
\frac{d}{d t}\langle\langle\langle\psi(t) \mid \psi(t)\rangle\rangle\rangle & =2 \gamma \sum_{i, j} \int_{0}^{t} D_{i j}(t, s)\left\langle\left\langle\left[\frac{\delta\langle\psi(t)|}{\delta w_{j}(s)}\right] A_{i} \mid \psi(t)\right\rangle\right\rangle d s \\
& +2 \gamma \sum_{i, j} \int_{0}^{t} D_{i j}(t, s)\left\langle\left\langle\left\langle\psi(t) \mid A_{i}\left[\frac{\delta|\psi(t)\rangle}{\delta w_{j}(s)}\right]\right\rangle d s \neq 0 .\right.\right.
\end{aligned}
$$

Since the time derivative of the average value of the square norm of the statevector is not zero, we have to add an extra term to equation (3.1), as expected and as it happens also in the case of white noise. Relation (3.8) tells us which kind of term must be added. The conclusion follows: with reference to our procedure, the request that $P_{\text {Cook }}[w(t)]$ correctly defines a probability distribution, i.e. that the average value of the square norm of the statevector $|\psi(t)\rangle$ is conserved, leads to the stochastic Schrödinger equation:

$$
\frac{d|\psi(t)\rangle}{d t}=\left[-\frac{i}{\hbar} H_{0}+\sum_{i} A_{i} w_{i}(t)-2 \gamma \sum_{i, j} A_{i} \int_{0}^{t} d s D_{i j}(t, s) \frac{\delta}{\delta w_{j}(s)}\right]|\psi(t)\rangle .
$$

This is the main result of this section. Note that an equation like (3.9) has been derived in references [11, 13] by following a different line of thought.

Some comments are appropriate:

- Equation (3.9) no longer describes a Markovian evolution for the statevector unless the correlation functions $D_{i j}(t, s)$ are Dirac- $\delta$ 's in the time variable - i.e. the stochastic processes $w_{i}(t)$ are white in time. As a consequence, the corresponding equation for the statistical operator is not of the quantum-dynamical-semigroup type [14, 15, contrary to what happen for the case of CSL (see equation 2.7)). 
- In general, the explicit form of the functional derivatives of $|\psi(t)\rangle$ with respect to the noise $w_{i}(t)$ cannot be evaluated exactly, except for few special cases, two of which will be considered in the next section. Therefore, in the general case it is difficult to analyze the time evolution of the statevector and the statistical properties of the ensemble of states generated by the stochastic processes. In particular, one cannot write a closed equation for the evolution of the statistical operator.

\section{Two special cases}

In order to understand the kind of difficulties one encounters when working with non-white stochastic processes, and in particular the reasons for which the functional derivative of the statevector $|\psi(t)\rangle$ in general cannot be computed exactly, let us reconsider equation (3.7), writing explicitly its perturbative expansion:

$$
\begin{aligned}
T e^{-\frac{i}{\hbar} H_{0} t} & +\sum_{i} A_{i} \int_{0}^{t} w_{i}(s) d s \\
& =\sum_{n=0}^{\infty}\left[-\frac{i}{\hbar}\right]^{n} \frac{1}{n !} \int_{0}^{t} d t_{1} \ldots \int_{0}^{t} d t_{n} T\left\{H\left(t_{1}\right) \ldots H\left(t_{n}\right)\right\}
\end{aligned}
$$

where we have defined the operator:

$$
H(t)=H_{0}+i \hbar \sum_{i} A_{i} w_{i}(t) .
$$

The functional derivative of $|\psi(t)\rangle$ with respect to $w_{j}(s)$ can be obtained deriving term by term the series (4.1). The derivative of the term $n=0$ is zero; the derivative of the term $n=1$ is:

$$
\frac{\delta}{\delta w_{j}(s)}\left[-\frac{i}{\hbar} \int_{0}^{t} d t_{1} H\left(t_{1}\right)\right]=-\frac{i}{\hbar} \int_{0}^{t} d t_{1}\left[i \hbar \delta\left(s-t_{1}\right) A_{j}\right]=A_{j} .
$$

The next $(n=2)$ term is:

$$
\left[-\frac{i}{\hbar}\right]^{2} \frac{1}{2} \int_{0}^{t} d t_{1} \int_{0}^{t} d t_{2} T\left\{H\left(t_{1}\right) H\left(t_{2}\right)\right\}
$$

The functional derivative of the time-ordered product $T\left\{H\left(t_{1}\right) H\left(t_{2}\right)\right\}=\theta\left(t_{1}-\right.$ $\left.t_{2}\right) H\left(t_{1}\right) H\left(t_{2}\right)+\theta\left(t_{2}-t_{1}\right) H\left(t_{2}\right) H\left(t_{1}\right)$ is:

$$
\begin{aligned}
& \frac{\delta}{\delta w_{j}(s)} T\left\{H\left(t_{1}\right) H\left(t_{2}\right)\right\}= \\
& \quad=i \hbar \theta\left(t_{1}-t_{2}\right)\left[\delta\left(t_{1}-s\right) A_{j} H\left(t_{2}\right)+\delta\left(t_{2}-s\right) H\left(t_{1}\right) A_{j}\right] \\
& \quad+i \hbar \theta\left(t_{2}-t_{1}\right)\left[\delta\left(t_{2}-s\right) A_{j} H\left(t_{1}\right)+\delta\left(t_{1}-s\right) H\left(t_{2}\right) A_{j}\right] .
\end{aligned}
$$

\footnotetext{
${ }^{4}$ We assume that the initial state $|\psi(0)\rangle$ does not depend on the stochastic processes $w_{i}(t)$.
} 
We note that the first and third terms at the right hand side of (4.5) differ only for the exchange of the dummy variables $t_{1} \leftrightarrow t_{2}$; the same is true for the second and the fourth term. The derivative of the $n=2$ term (i.e. of Eq. (4.4)) is then:

$$
A_{j}\left[-\frac{i}{\hbar} \int_{0}^{s} d t_{1} H\left(t_{1}\right)\right]+\left[-\frac{i}{\hbar} \int_{s}^{t} d t_{1} H\left(t_{1}\right)\right] A_{j} .
$$

Equation (4.6) does not have a simple form, contrary to 4.3), and derivatives of higher terms are more and more complicated, due to the fact that the operators $A_{j}$ in general do not commute with the Hamiltonian $H_{0}$. In fact, would they commute, equation (4.6) would simplify to:

$$
A_{j}\left[-\frac{i}{\hbar} \int_{0}^{t} d t_{1} H\left(t_{1}\right)\right]
$$

i.e. the derivative of the second term would give $A_{j}$ times the first term. Moreover, if $\left[A_{j}, H_{0}\right]=0$, the functional derivative of the term $n+1$ gives $A_{j}$ times the $n$-th term:

$$
\frac{\delta}{\delta w_{j}(s)}|\psi(t)\rangle \quad=\quad A_{j}|\psi(t)\rangle
$$

as we are going to prove. In fact, the hypothesis that the operators $A_{i}$ commute with the Hamiltonian $H_{0}$ is equivalent to the (more elegant) requirement that the operators $H(t)$ defined in (4.2) commute at different times. In this case, the time-ordered product in the exponential series (4.1) can be omitted, and the functional derivative of the $n$-th term is:

$$
\begin{aligned}
& \frac{\delta}{\delta w_{j}(s)}\left[-\frac{i}{\hbar}\right]^{n} \frac{1}{n !} \int_{0}^{t} d t_{1} \ldots \int_{0}^{t} d t_{n}\left\{H\left(t_{1}\right) \ldots H\left(t_{n}\right)\right\}= \\
& =\left[-\frac{i}{\hbar}\right]^{n} \frac{1}{n !} \sum_{i=1}^{n} \int_{0}^{t} d t_{1} \ldots \int_{0}^{t} d t_{n}\left\{H\left(t_{1}\right) \ldots \frac{\delta H\left(t_{i}\right)}{\delta w_{j}(s)} \ldots H\left(t_{n}\right)\right\}= \\
& =\left[-\frac{i}{\hbar}\right]^{n} \frac{1}{(n-1) !} \int_{0}^{t} d t_{1} \ldots \int_{0}^{t} d t_{n}\left\{\frac{\delta H\left(t_{1}\right)}{\delta w_{j}(s)} \ldots H\left(t_{n}\right)\right\}= \\
& =A_{j}\left[-\frac{i}{\hbar}\right]^{n-1} \frac{1}{(n-1) !} \int_{0}^{t} d t_{1} \ldots \int_{0}^{t} d t_{n-1}\left\{H\left(t_{1}\right) \ldots H\left(t_{n-1}\right)\right\} .
\end{aligned}
$$

This completes the proof. Note also that, when $s=t$, an extra factor $1 / 2$ appears in (4.8), because in this case the Dirac delta function arising from the functional derivative of $H(t)$ is centered in one of the two extreme points of the interval of integration.

Recently, S. Adler and P. Horwitz [17] (see also [18]) have proposed a whitenoise model of dynamical reductions in which the operators $A_{i}$ are taken to be 
functions of the Hamiltonian $H_{0}$; this implies that the stochastic terms of equation (2.1) drive the statevector into the energy eigenmanifolds of the physical system. Making such a choice in the non-white equation (3.9), the operators $H(t)$ at different times commute among themselves, the functional derivatives of the statevector $|\psi(t)\rangle$ can be computed, and equation (3.9) becomes:

$$
\frac{d|\psi(t)\rangle}{d t}=\left[-\frac{i}{\hbar} H_{0}+\sum_{i} A_{i} w_{i}(t)-2 \gamma \sum_{i, j} A_{i} A_{j} \int_{0}^{t} D_{i j}(t, s) d s\right]|\psi(t)\rangle,
$$

with $A_{i}=A_{i}\left(H_{0}\right)$. Equation 4.10 ) is exact and, correspondingly, one can easily derive a closed equation for the time evolution of the statistical operator. All the statistical properties concerning the physical system can be evaluated exactly.

We conclude the section showing that the functional derivatives of $|\psi(t)\rangle$ can be explicitly evaluated also in the case of general white noise stochastic processes, without having to require that $H_{0}$ commutes with $A_{i}$. Moreover, we will prove that in this case equation (3.9) reduces to (2.1), as expected.

Under the assumption of white-noise stochastic processes $\left(D_{i j}\left(t_{1}, t_{2}\right)=\right.$ $\left.\delta_{i j} \delta\left(t_{1}-t_{2}\right)\right)$, the Furutsu-Novikov relation

$$
\left\langle\left\langle F[w(t)] w_{i}(t)\right\rangle\right\rangle=\gamma\left\langle\left\langle\frac{\delta F[w(t)]}{\delta w_{i}(t)}\right\rangle\right\rangle
$$

leads to the following expression for the time derivative of the average value of the square norm of the statevector $|\psi(t)\rangle$ satisfying equation (3.1):

$$
\begin{aligned}
\frac{d}{d t}\langle\langle\langle\psi(t) \mid \psi(t)\rangle\rangle\rangle= & \left.2 \gamma \sum_{i}\left\langle\left\langle\left[\frac{\delta\langle\psi(t)|}{\delta w_{i}(t)}\right] A_{i} \mid \psi(t)\right\rangle\right\rangle\right\rangle+ \\
& 2 \gamma \sum_{i}\left\langle\left\langle\left\langle\psi(t) \mid A_{i}\left[\frac{\delta|\psi(t)\rangle}{\delta w_{i}(t)}\right]\right\rangle\right\rangle .\right.
\end{aligned}
$$

We now have to evaluate the functional derivatives of the statevector, taking into account that the noises $w_{i}(t)$ (appearing in the derivatives) are taken at time $t$.

The derivative of the term $n=1$ is equal to $(1 / 2) A_{j}$ (see equation (14.3)), the factor $(1 / 2)$ deriving from the Dirac delta function $\delta\left(t-t_{1}\right)$ which is integrated between 0 and $t$. For the derivative of the $n=2$ term, let us look at expression (4.6). If we take $s=t$, the second term goes to zero, while the first one givesf:

$$
\frac{1}{2} A_{j}\left[-\frac{i}{\hbar} \int_{0}^{t} d t_{1} H\left(t_{1}\right)\right] .
$$

\footnotetext{
${ }^{5}$ The factor $(1 / 2)$ appears for the same reason as before.
} 
In general, the functional derivative of any terms of the exponential series (4.1) gives $(1 / 2) A_{j}$ times the previous term, so that:

$$
\frac{\delta}{\delta w_{j}(t)}|\psi(t)\rangle=\frac{1}{2} A_{j}|\psi(t)\rangle
$$

This means that the square-norm-preserving Schrödinger equation is:

$$
\frac{d|\psi(t)\rangle}{d t}=\left[-\frac{i}{\hbar} H_{0}+\sum_{i} A_{i} w_{i}(t)-\gamma \sum_{i} A_{i} A_{j}\right]|\psi(t)\rangle,
$$

which coincides with the original CSL equation (2.1). An alternative and quicker way to derive the white-noise limit is to replace $D_{i j}(t, s)$ with $\delta_{i j} \delta(t-s)$ in equation (3.9) and to show that (4.14) is a consistent solution.

\section{The reduction mechanism}

Here, we will analyze under which conditions the new terms in the modified Schrödinger equation (3.9) induce, for large times, the reduction of the statevector to one of the common eigenstates of the commuting operators $A_{i}$.

For this purpose, let us disregard the Hamiltonian $H_{0}$; under this assumption the operators $H(t)$ commute at different times and (as discussed in the previous section) the functional derivatives of the statevector $|\psi(t)\rangle$ give the operators $A_{i}$ times $|\psi(t)\rangle$. Equation (3.9) becomes then:

$$
\frac{d|\psi(t)\rangle}{d t}=\left[\sum_{i} A_{i} w_{i}(t)-2 \gamma \sum_{i, j} A_{i} A_{j} \int_{t_{0}}^{t} D_{i j}(t, s) d s\right]|\psi(t)\rangle .
$$

The equation for the statistical operator can now be easily derived; using the definition (2.6), we get:

$$
\frac{d \rho(t)}{d t}=-\gamma \sum_{i, j}\left[A_{i},\left[A_{j}, \rho(t)\right]\right] \int_{t_{0}}^{t} D_{i j}(t, s) d s,
$$

which is a consistent generalization of the CSL equation (2.7) when the Hamiltonian $H_{0}$ is omitted: in fact, if the stochastic processes $w_{i}(t)$ are independent and white $\left(D_{i j}\left(t_{1}, t_{2}\right)=\delta_{i j} \delta\left(t_{1}-t_{2}\right)\right)$, then (5.2) reduces exactly to (2.7).

In order to test the reduction properties, we will show first of all how the reduction mechanism works for the statistical operator (see footnote 1). As in section 2 , let us suppose that the common eigenmanifolds of the operators $A_{i}$,

\footnotetext{
${ }^{6}$ Here and in what follows, we consider a generic initial time $t_{0}$.
} 
which we assume to have a purely discrete spectrum, are one-dimensional; let $|\alpha\rangle$ be the vector spanning the $\alpha$-eigenmanifold. The equation for the matrix elements $\langle\alpha|\rho(t)| \beta\rangle$ is:

$$
\frac{d\langle\alpha|\rho(t)| \beta\rangle}{d t}=-\gamma \sum_{i, j}\left(a_{i \alpha}-a_{i \beta}\right)\left(a_{j \alpha}-a_{j \beta}\right) \int_{t_{0}}^{t} D_{i j}(t, s) d s\langle\alpha|\rho(t)| \beta\rangle .
$$

Making use of the symmetry property of the correlation functions:

$$
D_{i j}\left(t_{1}, t_{2}\right)=D_{j i}\left(t_{2}, t_{1}\right),
$$

we can write the solution of equation (5.3) in the following form (see also [19]):

$$
\langle\alpha|\rho(t)| \beta\rangle=e^{-\frac{\gamma}{2} \sum_{i, j}\left(a_{i \alpha}-a_{i \beta}\right)\left(a_{j \alpha}-a_{j \beta}\right) \int_{t_{0}}^{t} d t_{1} \int_{t_{0}}^{t} d t_{2} D_{i j}\left(t_{1}, t_{2}\right)}\left\langle\alpha\left|\rho\left(t_{0}\right)\right| \beta\right\rangle .
$$

From equation (5.5), we sees that if $|\alpha\rangle=|\beta\rangle$, the exponent is zero: as in CSL, the diagonal elements of the density matrix do not change in time. If, on other other hand $|\alpha\rangle \neq|\beta\rangle$, the evolution of the matrix element depends on the time behavior the correlation functions $D_{i j}\left(t_{1}, t_{2}\right)$.

If we want the off-diagonal elements to be damped at large times, two conditions must be satisfied. The first one is that the exponent in (5.5) must be negative: this is always true, since the correlation function of a Gaussian process is positive definite.

The second condition is that the double integral of the correlation function must diverge for large times:

$$
\int_{t_{0}}^{t} d t_{1} \int_{t_{0}}^{t} d t_{2} D_{i j}\left(t_{1}, t_{2}\right) \longrightarrow+\infty \quad \text { for } t \rightarrow+\infty
$$

so that the off-diagonal elements of the density matrix go to zero. This condition is not a priori satisfied by a generic Gaussian stochastic field. At any rate, physical reasonable stochastic fields always satisfy it: here we present just a couple of meaningful examples.

Suppose the stochastic fields $w_{i}(t)$ are equal and independent, with a (normalized) Gaussian correlation function:

$$
D_{i j}\left(t_{1}, t_{2}\right)=\delta_{i j} \frac{1}{\sqrt{2 \pi} \tau} e^{-\frac{\left(t_{1}-t_{2}\right)^{2}}{2 \tau^{2}}} .
$$

Let us also take $t_{0}=-\infty$. Equation (5.3) then becomes:

$$
\frac{d\langle\alpha|\rho(t)| \beta\rangle}{d t}=-\frac{\gamma}{2} \sum_{i}\left(a_{i \alpha}-a_{i \beta}\right)^{2}\langle\alpha|\rho(t)| \beta\rangle,
$$


which is independent from the correlation time $\tau$, and moreover it corresponds exactly to the CSL equation (2.9). The fact that we have taken $t_{0}=-\infty$ means that the correspondence between equation $(5.3)$ - with a correlation function like (5.7) - and equation (2.9) is exact only in the limit of large times. Note also that if we take the limit $\tau \rightarrow 0$, the gaussian process becomes a white noise process with a Dirac $-\delta$ correlation function and we recover, again, the CSL theory.

As a second example, suppose the correlation function is:

$$
D_{i j}\left(t_{1}, t_{2}\right)=\delta_{i j} \frac{1}{2 \tau} e^{-\frac{\left|t_{1}-t_{2}\right|}{\tau}} .
$$

Equation (5.3) becomes:

$$
\frac{d\langle\alpha|\rho(t)| \beta\rangle}{d t}=-\frac{\gamma}{2}\left[1-e^{-\frac{\left(t-t_{0}\right)}{\tau}}\right] \sum_{i}\left(a_{i \alpha}-a_{i \beta}\right)^{2}\langle\alpha|\rho(t)| \beta\rangle .
$$

As before, the off-diagonal elements are exponentially damped and, in the limit $t \rightarrow+\infty$ we recover the behavior of CSL. Note that the effect of a non-white correlation function is that of decreasing the reduction rate of the localization mechanism.

We now analyze how the reduction mechanism works at the wavefunction level, proving in this way that equation (5.1) leads to the reduction of the statevector into one of the common eigenmanifolds of the operators $A_{i}$. As in reference [5], we consider a simplified dynamics in which only one operator $A$ appears in equation (5.1). This operator is coupled to a single stochastic process $w(t)$, whose correlation function is $D\left(t_{1}, t_{2}\right)$. Finally, we assume that at the initial time $t_{0}$ the statevector is:

$$
\left|\psi\left(t_{0}\right)\right\rangle=P_{\alpha}\left|\psi\left(t_{0}\right)\right\rangle+P_{\beta}\left|\psi\left(t_{0}\right)\right\rangle,
$$

where $P_{\alpha}$ and $P_{\beta}$ are projection operators onto the eigenmanifolds of $A$ corresponding to two different eigenvalues $\alpha$ and $\beta$, respectively. The solution of equation (5.1) is:

$$
|\psi(t)\rangle=e^{\alpha x(t)-\alpha^{2} \gamma f(t)} P_{\alpha}\left|\psi\left(t_{0}\right)\right\rangle+e^{\beta x(t)-\beta^{2} \gamma f(t)} P_{\alpha}\left|\psi\left(t_{0}\right)\right\rangle,
$$

where

$$
x(t)=\int_{t_{0}}^{t} w(s) d s, \quad f(t)=\int_{t_{0}}^{t} d s_{1} \int_{t_{0}}^{t} d s_{2} D\left(s_{1}, s_{2}\right) .
$$


Note that $\gamma f(t)=\left\langle\left\langle x^{2}(t)\right\rangle\right.$, i.e. such a quantity is the variance of the stochastic process $x(t)$.

Since the "raw" probability distribution of the process $x(t)$ is:

$$
P_{\text {Raw }}[x(t)]=\frac{1}{\sqrt{2 \pi \gamma f(t)}} e^{-\frac{1}{2 \gamma f(t)} x^{2}(t)},
$$

taking into account the cooking prescription (3.4) we obtain:

$$
\begin{aligned}
P_{\text {Cook }}[x(t)] & =\| P_{\alpha}\left|\psi\left(t_{0}\right)\right\rangle \|^{2} \frac{1}{\sqrt{2 \pi \gamma f(t)}} e^{-\frac{1}{2 \gamma f(t)}[x(t)-2 \alpha \gamma f(t)]^{2}} \\
& +\| P_{\beta}\left|\psi\left(t_{0}\right)\right\rangle \|^{2} \frac{1}{\sqrt{2 \pi \gamma f(t)}} e^{-\frac{1}{2 \gamma f(t)}[x(t)-2 \beta \gamma f(t)]^{2}} .
\end{aligned}
$$

Equation (5.15) implies that, if $f(t) \rightarrow+\infty$ when $t \rightarrow+\infty$, the stochastic process $x(t)$ will take either a value close to $2 \alpha \gamma f(t)$ - within an interval of width $\sqrt{\gamma f(t)}$ - or a value close to $2 \beta \gamma f(t)$, within the same interval|]. Of course, the requirement that $f(t) \rightarrow+\infty$ as time increases is exactly the same as requirement (5.6) which guarantees the damping of the off-diagonal elements of the density matrix.

Suppose now that the actual realization of the stochastic process $x(t)$ occurs around $2 \alpha \gamma f(t)$; the corresponding probability is $\| P_{\alpha}|\psi(0)\rangle \|^{2}$. We then have:

$$
\frac{\| P_{\beta}|\psi(t)\rangle \|^{2}}{\| P_{\alpha}|\psi(t)\rangle \|^{2}} \simeq e^{-2 \gamma(\alpha-\beta)^{2} f(t)} \frac{\| P_{\beta}|\psi(0)\rangle \|^{2}}{\| P_{\alpha}|\psi(0)\rangle \|^{2}} \rightarrow 0 \quad \text { as } t \rightarrow \infty
$$

which means that the statevector $|\psi(t)\rangle$ is driven into the eigenmanifold of the operator $A$ corresponding to the eigenvalue $\alpha$. By the same reasoning, it is immediate to see that, with a probability equal to $\| P_{\beta}|\psi(0)\rangle \|^{2}$, the statevector is driven into the eigenmanifold associated to the eigenvalue $\beta$. We have thus proved that the statevector $|\psi(t)\rangle$ undergoes a random spontaneous localization into one of the two eigenmanifolds of the operator $A$, with a probability which coincides with the one assigned by standard Quantum Mechanics to the outcomes of an experiment aimed to measure the observable $A$.

${ }^{7}$ As noted in [5], even though the interval $\sqrt{\gamma f(t)}$ tends to infinity as time increases, the ratio $\sqrt{\gamma f(t)} / 2(\alpha-\beta) \gamma f(t)$ goes to zero. 


\section{The average value of observables}

When one disregards the Hamiltonian term $H_{0}$, it is not difficult to see how the stochastic terms affect the average value of physical quantities.

The mean value of an operator $O$ (for simplicity, we consider an observable which does not depend explicitly on time) is defined as the expectation value $\langle\phi(t)|O| \phi(t)\rangle$, averaged over all possible realizations of the stochastic noises:

$$
\begin{aligned}
\langle O\rangle & =\int \mathcal{D}[w(t)]\langle\psi(t)|O| \psi(t)\rangle P_{\mathrm{Cook}}[w(t)]= \\
& =\langle\langle\langle\psi(t)|O| \psi(t)\rangle\rangle\rangle .
\end{aligned}
$$

Its time derivative can be calculated following almost the same steps which, in the previous section, have led to equation (5.2) for statistical operator; the final equation is:

$$
\frac{d\langle O\rangle}{d t}=-\gamma \sum_{i, j}\left\langle\left\langle\left\langle\psi(t)\left|\left[A_{i},\left[A_{j}, O\right]\right]\right| \psi(t)\right\rangle\right\rangle\right\rangle \int_{t_{0}}^{t} D_{i j}(t, s) d s,
$$

to be compared with the corresponding CSL-white noise equation:

$$
\frac{d\langle O\rangle}{d t}=-\frac{\gamma}{2} \sum_{i}\left\langle\left\langle\left\langle\psi(t)\left|\left[A_{i},\left[A_{i}, O\right]\right]\right| \psi(t)\right\rangle\right\rangle\right\rangle .
$$

The analysis of the previous section should have made clear how 6.1) differs from (6.2), so we will not repeat it here.

\section{Connection with CSL}

We now apply the formalism introduced in the previous sections to derive an equation with the property of localizing macroscopic systems in space, like in CSL; in other words, we specify the choice of the "preferred basis" $\left\{A_{i}\right\}$ in such a way to have a physically meaningful theory for our purposes.

The most natural choice for the operators $A_{i}$ is the number density operator for a system of identical particles:

$$
A_{i} \quad \longrightarrow \quad N(\mathbf{x})=\sum_{s} a^{\dagger}(\mathbf{x}, s) a(\mathbf{x}, s) .
$$

Correspondingly, the noises $w_{i}(t)$ are replaced by a stochastic field $w(\mathbf{x}, t)$, whose correlation function is $D\left(\mathbf{x}, t_{1} ; \mathbf{y}, t_{2}\right)$.

In reference [4], the transformation and invariance properties of dynamical reduction models have been discussed in detail. In particular, it has been 
proved that, in order for the physical properties of the model to be invariant under Galilean transformations (we speak of stochastic Galilean invariance), the correlation function $D\left(\mathbf{x}, t_{1} ; \mathbf{y}, t_{2}\right)$ itself must be invariant under the considered group of transformations, i.e.

$$
D\left(\mathbf{x}, t_{1} ; \mathbf{y}, t_{2}\right)=D\left(|\mathbf{x}-\mathbf{y}|, t_{1}-t_{2}\right)
$$

the easiest way to construct a function like $(7.2)$ is to take the product of two functions of the space and time variables, respectively:

$$
D\left(\mathbf{x}, t_{1} ; \mathbf{y}, t_{2}\right)=g(|\mathbf{x}-\mathbf{y}|) h\left(t_{1}-t_{2}\right) .
$$

As regards $g(|\mathbf{x}-\mathbf{y}|)$, a reasonable choice is a gaussian function, like in CSL:

$$
g(|\mathbf{x}-\mathbf{y}|)=\gamma\left(\frac{\alpha}{4 \pi}\right)^{\frac{3}{2}} e^{-\frac{\alpha}{4}(\mathbf{x}-\mathbf{y})^{2}}
$$

with $1 / \sqrt{\alpha} \simeq 10^{-5} \mathrm{~cm}$.

It is natural to choose a gaussian function also for $h\left(t_{1}-t_{2}\right)$ :

$$
h\left(t_{1}-t_{2}\right)=\left(\frac{\beta}{4 \pi}\right)^{\frac{1}{2}} e^{-\frac{\beta}{4}\left(t_{1}-t_{2}\right)^{2}} .
$$

With the above choice, we have introduced a new parameter $(\beta)$; this can be considered as a drawback of the model. However, we note that it always is possible to define $\beta$ in terms of $\alpha, \gamma$ and fundamental constants of nature, so that no new arbitrary parameter is introduced into the model. As an example, we can choose $\beta=c^{2} \alpha \simeq 10^{30} \mathrm{sec}^{-2}$, where $c$ is the speed of light. This choice is particularly appropriate in the light of a possible relativistic generalization of the theory, which we will discuss in a future paper. Moreover, such a choice corresponds to an extremely small correlation time, so that for ordinary systems (moving slower than the speed of light) the behavior of the model is similar to the one deriving from the white-noise CSL.

The modified equation (3.9) for the statevector evolution becomes now:

$$
\begin{aligned}
\frac{d|\psi(t)\rangle}{d t}= & {\left[-\frac{i}{\hbar} H_{0}+\int d^{3} x N(\mathbf{x}) w(\mathbf{x}, t)-\right.} \\
& \left.-2 \gamma \int d^{3} x d^{3} y N(\mathbf{x}) g(|\mathbf{x}-\mathbf{y}|) \int_{t_{0}}^{t} d s h(t-s) \frac{\delta}{\delta w(\mathbf{y}, s)}\right]|\psi(t)\rangle .
\end{aligned}
$$

If we ignore the free Hamiltonian $H_{0}$, i.e. we confine our considerations to the 
reduction mechanism 8 , equation $(7.6)$ becomes:

$$
\frac{d|\psi(t)\rangle}{d t}=\left[\int d^{3} x N(\mathbf{x}) w(\mathbf{x}, t)-\gamma(t) \int d^{3} x d^{3} y N(\mathbf{x}) g(|\mathbf{x}-\mathbf{y}|) N(\mathbf{y})\right]|\psi(t)\rangle
$$

with:

$$
\gamma(t)=2 \gamma \int_{t_{0}}^{t} d s h(t-s) .
$$

The corresponding equation for the statistical operator is:

$$
\frac{d}{d t} \rho(t)=-\frac{\gamma(t)}{2} \int d^{3} x d^{3} y[N(\mathbf{x}),[N(\mathbf{y}), \rho(t)]] g(|\mathbf{x}-\mathbf{y}|) .
$$

Equation (7.6) can be rewritten in a form closer to equation (2.12), which will be useful for the subsequent discussion. Let us define a new Gaussian stochastic process $\bar{w}(\mathbf{x}, t)$, which is connected to $w(\mathbf{x}, t)$ by the relation:

$$
w(\mathbf{x}, t)=\left(\frac{\alpha}{2 \pi}\right)^{\frac{3}{2}} \int d^{3} x e^{-\frac{\alpha}{2}(\mathbf{x}-\mathbf{y})^{2}} \bar{w}(\mathbf{y}, t) .
$$

$\bar{w}(\mathbf{x}, t)$ has zero mean, and correlation function

$$
\left\langle\left\langle\bar{w}\left(\mathbf{x}, t_{1}\right) \bar{w}\left(\mathbf{y}, t_{2}\right)\right\rangle\right\rangle=\gamma \delta^{(3)}(\mathbf{x}-\mathbf{y}) h\left(t_{1}-t_{2}\right) .
$$

Using the following relation:

$$
\begin{aligned}
\frac{\delta}{\delta \bar{w}(\mathbf{x}, s)}|\psi(t)\rangle & =\int d^{3} y \frac{\delta w(\mathbf{y}, s)}{\delta \bar{w}(\mathbf{x}, s)} \frac{\delta}{\delta w(\mathbf{y}, s)}|\psi(t)\rangle= \\
& =\left(\frac{\alpha}{2 \pi}\right)^{\frac{3}{2}} \int d^{3} y e^{-\frac{\alpha}{2}(\mathbf{x}-\mathbf{y})^{2}} \frac{\delta}{\delta w(\mathbf{y}, s)}|\psi(t)\rangle,
\end{aligned}
$$

it can be easily seen that (7.6) is equivalent to the equation:

$$
\begin{aligned}
\frac{d|\psi(t)\rangle}{d t}= & {\left[-\frac{i}{\hbar} H_{0}+\int d^{3} x \mathcal{N}(\mathbf{x}) w(\mathbf{x}, t)-\right.} \\
& \left.-2 \gamma \int d^{3} x \mathcal{N}(\mathbf{x}) \int_{t_{0}}^{t} d s h(t-s) \frac{\delta}{\delta \bar{w}(\mathbf{x}, s)}\right]|\psi(t)\rangle,
\end{aligned}
$$

with $\mathcal{N}(\mathbf{x})$ defined by $(2.10)$.

\footnotetext{
${ }^{8}$ For the physically interesting cases, e.g. for the dynamical evolution of macrosystems, such an assumption is justified by the fact that the effect of the reduction is much faster that the tipical times in which the Hamiltonian can induce appreciable dynamical changes of the statevector.
} 


\subsection{Dynamics for macroscopic rigid bodies}

As for white-noise CSL [3, it is not difficult to discuss the physical implications of equation (7.6) - or equation (7.13) - for the case of a macroscopic rigid body, i.e. a body such that the wavefunctions of its constituents can be considered very well localized with respect to the localization length $1 / \sqrt{\alpha}$.

To be precise, in analogy with the procedure followed in [3], let us consider a system of $N$ identical particles of coordinates $\mathbf{q}_{i}$; let

$$
\mathbf{Q}=\frac{1}{N} \sum_{i=1}^{N} \mathbf{q}_{i}
$$

be the center of mass coordinate, and let us write

$$
\mathbf{q}_{i}=\mathbf{Q}+\overline{\mathbf{q}}_{i}
$$

where the coordinates $\overline{\mathbf{q}}_{i}$ are functions of $3 N-3$ independent internal variables 9 , which we call $r$. Let us consider the wavefunction

$$
|\psi(q, s)\rangle=|\phi(\mathbf{Q})\rangle|\varphi(r, s)\rangle \quad|\varphi(r, s)\rangle=\left[\begin{array}{c}
A \\
S
\end{array}\right]|\Delta(r, s)\rangle,
$$

where $q=\left\{\mathbf{q}_{i}\right\}$ and $s=\left\{s_{i}\right\}$ are the sets of the space and spin coordinates of the $N$ particles, respectively, while "A" and "S" mean symmetrization or antisymmetrization with respect to the interchange of the variables $\left(\mathbf{q}_{i}, s_{i}\right)$, respectively.

In reference [3] it has been proved that if the wavefunction of the internal degrees of freedom is very well peaked with respect to the characteristic length $1 / \sqrt{\alpha}$, then, to an extremely high degree of accuracy,

$$
\mathcal{N}(\mathbf{x})|\phi(\mathbf{Q})\rangle|\varphi(r, s)\rangle \quad=\quad F(\mathbf{Q}-\mathbf{x})|\phi(\mathbf{Q})\rangle|\varphi(r, s)\rangle,
$$

with

$$
F(\mathbf{Q}-\mathbf{x})=\left(\frac{\alpha}{2 \pi}\right)^{\frac{3}{2}} \sum_{i=1}^{N} e^{-\frac{\alpha}{2}\left[\mathbf{Q}+\overline{\mathbf{q}}_{i}\left(r_{0}\right)-\mathbf{x}\right]^{2},},
$$

where $r_{0}$ describes the set of the average equilibrium positions of the particles of the rigid body. Equation (7.18) means that the operators $\mathcal{N}(\mathbf{x})$ act only on the center of mass wavefunction $|\phi(\mathbf{Q})\rangle$.

As a consequence, if the Hamiltonian $H_{0}$ can be written as

$$
H_{0}=H_{\mathbf{Q}}+H_{r}
$$

\footnotetext{
${ }^{9}$ See ref. [3 for further details on the degrees of freedom of the system.
} 
and if $|\phi(\mathbf{Q})\rangle$ and $|\varphi(r, s)\rangle$ satisfy the equations

$$
\begin{aligned}
\frac{d|\phi(\mathbf{Q}, t)\rangle}{d t}= & {\left[-\frac{i}{\hbar} H_{\mathbf{Q}}+\int d^{3} x \mathcal{N}(\mathbf{x}) \bar{w}(\mathbf{x}, t)-\right.} \\
& \left.-2 \gamma \int d^{3} x \mathcal{N}(\mathbf{x}) \int_{t_{0}}^{t} d s h(t-s) \frac{\delta}{\delta \bar{w}(\mathbf{x}, s)}\right]|\phi(\mathbf{Q}, t)\rangle \\
\frac{d|\varphi(r, s, t)\rangle}{d t}= & {\left[-\frac{i}{\hbar} H_{r}\right]|\varphi(r, s, t)\rangle }
\end{aligned}
$$

then $|\psi(q, s, t)\rangle$ satisfies equation (7.13) or, equivalently, equation (7.6).

Equations (7.20) and (7.21) imply that the center of mass and internal motion decouple, and that the stochastic terms affect only the center of mass and not the internal structure, as it happens for CSL.

Following the same arguments of reference [3], it can also be proven that the localization rate of the center of mass wavefunction grows linearly with the number of particles of the rigid body. Such a localization rate can be easily computed by studying the time evolution of the off-diagonal elements $\left\langle\mathbf{Q}^{\prime}\left|\rho_{\mathbf{Q}}\right| \mathbf{Q}^{\prime \prime}\right\rangle$ of the reduced statistical operator describing the center of mass motion of the system. As we did in section 5, we disregard the Hamiltonian $H_{\mathbf{Q}}$, in accordance with the fact that, for $\left|\mathbf{Q}^{\prime}-\mathbf{Q}^{\prime \prime}\right|>1 / \sqrt{\alpha}$, the reduction rate turns out to be much faster than the typical times required in order that the standard Schrödinger evolution induces appreciable changes of $|\phi(\mathbf{Q}, t)\rangle$. Under this assumption, equation (7.20) becomes:

$$
\frac{d|\phi(\mathbf{Q}, t)\rangle}{d t}=\left[\int d^{3} x F(\mathbf{Q}-\mathbf{x}) \bar{w}(\mathbf{x}, t)-\gamma(t) \int d^{3} x F^{2}(\mathbf{Q}-\mathbf{x})\right]|\phi(\mathbf{Q}, t)\rangle,
$$

and the corresponding equation for the matrix elements $\left\langle\mathbf{Q}^{\prime}\left|\rho_{\mathbf{Q}}\right| \mathbf{Q}^{\prime \prime}\right\rangle$ is:

$$
\frac{d\left\langle\mathbf{Q}^{\prime}\left|\rho_{\mathbf{Q}}(t)\right| \mathbf{Q}^{\prime \prime}\right\rangle}{d t}=-\Gamma\left(\mathbf{Q}^{\prime}, \mathbf{Q}^{\prime \prime}, t\right)\left\langle\mathbf{Q}^{\prime}\left|\rho_{\mathbf{Q}}(t)\right| \mathbf{Q}^{\prime \prime}\right\rangle
$$

with

$$
\begin{aligned}
\Gamma\left(\mathbf{Q}^{\prime}, \mathbf{Q}^{\prime \prime}, t\right)= & \gamma(t) \int d^{3} x\left[\frac{1}{2} F^{2}\left(\mathbf{Q}^{\prime}-\mathbf{x}\right)+\frac{1}{2} F^{2}\left(\mathbf{Q}^{\prime \prime}-\mathbf{x}\right)-\right. \\
& \left.F\left(\mathbf{Q}^{\prime}-\mathbf{x}\right) F\left(\mathbf{Q}^{\prime \prime}-\mathbf{x}\right)\right] .
\end{aligned}
$$

This is the same term as the one appearing in CSL, with $\gamma(t)$ replacing $\gamma$; this proves that also in our model the reduction frequency of the center of mass of the system grows linearly with the number of its constituents. Moreover, taking a large value for $\beta$, as it has been suggested previously, $\gamma(t) \rightarrow \gamma$ in very short times, so that the reducing dynamics is practically the same as the one of CSL. 


\section{Acknowledgements}

The authors would like to thank S. Adler for useful discussion and advices.

\section{References}

[1] G.C. Ghirardi, A. Rimini and T. Weber, Phys. Rev. D 34, 470 (1986).

[2] P. Pearle, Phys. Rev. A 39, 2277 (1989).

[3] G.C. Ghirardi, P. Pearle and A. Rimini, Phys. Rev. A 42, 78 (1990).

[4] G.C. Ghirardi, R. Grassi and P. Pearle, Found. Phys. 20, 1271 (1990).

[5] G.C. Ghirardi, R. Grassi and F. Benatti, Found. Phys. 25, 5 (1995).

[6] L. Diósi, Phys. Lett. A 129, 419 (1988).

[7] N. Gisin and I.C. Percival, Journ. Phys. A 25, 5677 (1992).

[8] P. Pearle, in: "Perspectives on Quantum Reality", R. Clifton ed., Kluwer Dordrecht, p. 93 (1996).

[9] P. Pearle, in: "Open Systems and Measurement in Relativistic Quantum Theory", F. Petruccione and H.P. Breuer eds., Springer Verlag, p. 195 (1999).

[10] L. Diósi, Phys. Rev. A 42, 5086 (1990).

[11] L. Diósi and W. T. Strunz, Phys. Lett. A 235, 569 (1997).

[12] G.C. Ghirardi and R. Grassi, in: Nuovi problemi della logica e della filosofia della scienza, CLUEB, Bologna (1991).

[13] A.A. Budini, Phys. Rev. A 63, 012106 (2000).

[14] G. Lindblad, Commun. Math. Phys. 48, 119 (1976).

[15] F. Benatti, G.C. Ghirardi, A. Rimini, T. Weber, Nuovo Cimento 101 B, 333 (1988).

[16] K. Sobczyk: "Stochastic Wave Propagation", Elsevier, Amsterdam (1985).

[17] S. Adler and P. Howritz: Journ. Math. Phys. 41, 2485 (2000). 
[18] S. Adler and T. Brun: Journ. Phys. A 34, 4797 (2001).

[19] L. Diósi, N. Gisin and W.T. Strunz, Phys. Rev. A 58, 1699 (1998). 